\title{
Neurofeedback y Trauma Craneoencefálico Frontal: Estudio de CAso ${ }^{1}$
}

\author{
Neurofeedback and Frontal Cranioencefalic Trauma: \\ Case Study
}

\author{
José Alberto Luna Hernández², Isabel Hernández Arteaga ${ }^{3}$, \\ Richard Alejandro Santander Araujo ${ }^{4}$
}

Recibido: 2019-02-15 Aceptado: 2019-04-21

Resumen: Este artículo presenta los resultados de la investigación sobre el tema de Trauma Craneoencefálico (TCE), una lesión adquirida que genera cambios a nivel cognitivo funcional y emocional. Objetivo: comprobar la efectividad del programa de rehabilitación atencional mediante neurofeedback, en paciente con TCE leve frontal. Metodología: estudio preexperimental con paciente de 29 años, a quien se aplicó evaluación pretratamiento, se intervino con 12 sesiones de neurofeedback y se evaluó postratamiento; estudio empírico-analítico de tipo explicativo. Resultados: indicaron cambios significativos en procesos principalmente atencionales. Conclusiones: se comprobó efectividad del programa, comparando datos pre y post-tratamiento, analizados para medir la magnitud del cambio terapéutico, indicando nivel confiable de efectividad; de igual manera se evidenció alcances importantes del efecto positivo en procesos de memoria y funciones ejecutivas.

Palabras clave: atención, funciones ejecutivas, memoria, neurofeedback, trauma craneoencefálico.

\begin{abstract}
This article presents the results of research on the subject of Cranioencephalic Trauma (TCE), an acquired lesion that generates changes at the functional and emotional cognitive level. Objective: to check the effectiveness of the attentional rehabilitation program by neurofeedback, in patients with light frontal CTE. Methodology: pre-experimental study with 29-year-old patient, to whom
\end{abstract}

Para citar este artículo en APA: Luna, J., Hernández, I y Santander, R. (2019). Neurofeedback y trauma craneoencefálico frontal: Estudio de caso. Revista de Psicologia Universidad de Antioquia, 11(1), 177-194. DOI: https://doi.org/10.17533/udea. rp.v11n1a08
Artículo original de caso único, derivado de la investigación Efectividad de un programa de neurofeedback en paciente con trauma craneoencefálico frontal leve. Programa de Psicología Universidad Mariana, Pasto, Colombia.

2 Magister en Neuropsicología Clínica, Especialista en Pruebas Neuropsicológicas. Docente Investigador y Coordinador del Laboratorio de Psicología de la Universidad Mariana. Correo: jluna@umarina.edu.co; https://orcid.org/0000-0003-24365218

3 Posdoctora en Investigación Educativa, Doctora en Ciencias de la Educación. Docente Investigadora de Posgrados de la Facultad de Educación de la Universidad Cooperativa de Colombia, sede Bogotá; http://orcid.org/0000-0002-62990236

4 Psicólogo; https://orcid.org/0000-0002-4010-4299 
pretreatment evaluation was applied, 12 neurofeedback sessions were intervened, and post-treatment was evaluated; empirical-analytical explanatory study. Results: indicated significant changes in mainly attentional processes. Conclusions: the effectiveness of the program was found, comparing pre- and post-treatment data, analyzed to measure the magnitude of therapeutic change, indi- cating reliable level of effectiveness; important scopes of the positive effect on memory processes and executive functions were also evident.

Keywords: Attention, executive functions, memory, neurofeedback, craniocerebral trauma.

\section{Introducción}

El Trauma Craneoencefálico (TCE) constituye una de las principales causas que alteran la fisiología y en algunos casos daños en la neuroanatomía del sistema nervioso central (SNC), se genera por golpes y/o movimientos bruscos; esto a su vez, tiene grandes repercusiones en varios componentes, incluyendo el cognitivo, emocional y conductual, reduciendo sus habilidades adaptativas y dificultando su interacción con el entorno (Guzmán, 2008; Juárez y Machinskaya, 2013).

Las alteraciones cognitivas ocasionadas por el TCE dificultan a las personas enfrentar los desafíos de la cotidianidad, además de presentar algunas limitaciones en el aprendizaje, las cuales truncan aún más su adaptabilidad y pueden repercutir en enfermedades neurocognitivas a largo plazo, en caso de no realizarse una adecuada intervención (Bernal, Vega y Hernández, 2009). Una de las principales causas del TCE son los accidentes de tránsito, los cuales producen la muerte de alrededor de 1,25 millones de personas, y cerca de 20 millones de casos, sufren traumatismos no mortales (OMS, 2016). Dicho flagelo tiene una incidencia mayor en la población joven que se encuentra entre los 15 y 30 años (Quijano, Arango, Cuervo y Aponte, 2012; Donéstevez, Cañizares, Alba y Alegret, 2017; OMS, 2016).

Ante estas cifras y el desafío cotidiano del siglo XXI, se llama la atención sobre la importancia de aplicar nuevas estrategias tecnológicas que den evidencia empírica, con resultados objetivamente fiables en la evaluación e intervención; siendo el neurofeedback una herramienta alternativa para la solución de esta problemática; ante lo cual, en la investigación objeto de este artículo se examinaron los resultados obtenidos con neurofeedback, dirigido a procesos atencionales para comprobar su efectividad (Méndez y Ordoñez, 2012). 
Si bien se han demostrado efectos contundentes y precisos al aplicar la herramienta en otro tipo de déficits, los casos de pacientes con TCE frontal leve, no son la excepción; estudios investigativos proponen a esta herramienta como una opción de intervención cognitiva en este tipo de casos; lo que sugiere la necesidad de comprobar científicamente su efectividad terapéutica (Stokes \& Lappin, 2010; Young, Tae \& Hwa, 2015; Barrera, Gómez y Prieto, 2013; Bonilla, González, Amaya, Ríos y Bonilla, 2016). De comprobarse como efectivo el uso del neurofeedback, permitiría mejorar el desempeño y la funcionalidad de la persona afectada (Machuca, León y Barroso, 2006).

El presente caso, se dirigió principalmente a rehabilitar la función atencional de un paciente con TCE leve a nivel frontal; este proceso psicológico se evidenció como el más afectado después de la evaluación neuropsicológica previa. En concordancia, Lubrini, Periáñez y Ríos (2009) afirman que la rehabilitación atencional viabiliza la recuperación funcional y cognitiva, dada su amplia locación neuroanatómica a nivel encefálico y su función facilitadora de las demás funciones cognitivas. De esta manera, la comprobación de la efectividad mediante técnicas vanguardistas de rehabilitación es pertinente e imperativa para permitir una estimulación cognitiva adecuada, enfocada a las necesidades propias del paciente; en este caso, a nivel atencional (Fernández, Bringas, Salazar, Rodríguez, García \& Torres, 2012).

Lo anterior permite visibilizar la necesidad de aplicar este tipo de herramientas para generar una recuperación ágil y eficaz de las dificultades cognitivas, las que se manifiestan debido a la lesión (Cuervo, Rincón y Quijano, 2009), tal como se evidencia en algunos resultados de investigaciones de autores como Stokes \& Lappin (2010), quienes en sus resultados refieren que el $70 \%$ de la población reportó una reducción de la sintomatología que aquejaba a pacientes con migraña, mediante el uso del neurofeedback.

Por su parte, Kianbakht, Naghel, Alidadi, Nejati, Kohandel, Ansarini \& Namdari (2015) manifiestan que su grupo de estudio con entrenamiento en neurofeedback mostró mejores resultados que el grupo control, argumentando que esta herramienta genera cambios en áreas de la corteza encargadas de la autorregulación y provoca cambios en las ondas cerebrales que permitan a los 
pacientes aprender a concentrarse, teniendo mejores mecanismos de autorregulación en sus procesos atencionales y velocidad de procesamiento.

\section{Descripción del caso}

Paciente masculino de 29 años, diestro, con escolaridad de pregrado en psicología, durante el tratamiento adelantaba principalmente estudios posgraduales y trabajaba de manera esporádica en el campo de seguridad y salud de trabajadores de la construcción. Sufrió una lesión (TCE leve) focal a nivel de la zona dorsolateral del lóbulo frontal izquierdo, consecuencia de un accidente de tránsito; no presentó fractura o depresión ósea; se generó hematoma epidural y como efecto patológico a daño secundario se presentó presión intracraneal (PIC) en esta zona. Se requirió intervención quirúrgica que consistió en un drenaje epidural.

Dicha afección se evidenció durante el periodo crítico mediante exploración neurológica, a través de resonancia magnética (RMN) y tomografía axial computarizada (TAC), realizada tres meses después del accidente. Posterior a los tres meses de recuperación se realiza una evaluación neuropsicológica pretratamiento (pre); luego, por decisión del equipo profesional (neuropsicología y psicología) y con soporte de consentimiento informado del paciente, se decide intervenir, haciendo uso del neurofeedback; medida tomada con base en las características del paciente y los resultados de la evaluación neuropsicológica, los cuales evidencian compromiso frontal relacionado principalmente con atención y memoria de trabajo, visible en los puntajes obtenidos en las pruebas (NEUROPSI atención y memoria y la Escala de Inteligencia para Adultos de Wechsler WAIS IV).

El presente artículo desarrolla como objetivo principal la comprobación de los beneficios del neurofeedback en paciente con un TCE frontal leve, para posteriormente conocer el impacto terapéutico de la herramienta (Young et al, 2015). 


\section{Descripción del proceso}

El programa de intervención se estructuró con base en el rastreo cognitivo previo al tratamiento, se plantearon cuatro fases. A saber, primera fase, corresponde a la evaluación neuropsicológica, pretratamiento; se utilizó los instrumentos NEUROPSI atención y memoria y la escala de inteligencia para adultos Wechsler WAIS IV, los cuales permitieron identificar y determinar el desempeño funcional de los procesos cognitivos del paciente (Bárcena et al, 2006; Juárez y Manchiskaya, 2013; Méndez, 2011; Barrera, Gómez y Prieto, 2013; Barrero, García y Ojeda, 2005). Durante esta evaluación, desde la perspectiva neuropsicológica se evidenció a nivel cognitivo que el proceso con mayor compromiso es a nivel atencional, diagnóstico que corresponde a déficit atencional secundario a TCE leve; esto disminuyó parte de la funcionalidad del paciente, porque al no prestar atención de forma adecuada, hace que tenga olvidos frecuentes, problemas con la retención de información, disminución de la velocidad de procesamiento de información y distracción (Fernández et al, 2012).

Segunda fase, enfocada al diseño e implementación del programa, el cual se diseñó alrededor del uso de la herramienta neurofeedback, específicamente mediante los módulos o "neurogames", correspondientes a juegos que interactúan o funcionan dependiendo de la actividad eléctrica cerebral, que estimulan la producción de ondas "BETHA" en los lóbulos frontales del paciente; dichas ondas se relacionan estrechamente con la función atencional (Camelo, Rojas, Mejía y Castro, 2015).

Se tuvo como premisa que este tipo de estimulación, permite que el paciente alcance oscilaciones bioeléctricas "adecuadas", en este caso principalmente a nivel frontal, que le permitan soportar los procesos complejos como el sostenimiento atencional o la memoria; así, al interactuar con la herramienta, el paciente debía alcanzar el rango BETHA de oscilación bioeléctrica, el cual está relacionado con ondas poco amplias y muy frecuentes que permiten el funcionamiento adecuado de los procesos atencionales, esto permitió generar retroalimentación de dicho procesos, para posteriormente lograr que el paciente sea consciente y autónomo frente al ajuste de su actividad cerebral 
y que a través de la auto-regulación y la retroalimentación, logre aprender a concentrarse y a mejorar sus procesos de tipo atencional (Kianbakht et al., 2015), tales como atención selectiva, sostenida, dividida, control atencional, retención de información y funciones asociadas como velocidad de procesamiento e información.

Por tanto, la herramienta neurofeedback se utilizó durante 12 sesiones intensivas, se programó y desarrollo dos sesiones por semana; en cada una de las sesiones se realizó de 3 a 4 entrenamientos previos, de dos minutos cada uno y por cada módulo (juegos o neurogames) de los cinco que contempla esta herramienta. Posterior al entrenamiento, el paciente interactuó con cada módulo durante cinco minutos, tiempo que es el estimado por defecto en cada módulo, esto se tomó como referente para evaluar su progreso; cabe resaltar que, cada módulo se contempla en niveles de dificultad que permitieron al paciente un mayor nivel de exigencia, por tal motivo, se realizaron entrenamientos previos con tiempos cortos. Para obtener los resultados finales, las dos primeras sesiones se tomaron como línea base para su respectivo análisis estadístico de caso único.

Tercera fase, corresponde a la evaluación neuropsicológica postratamiento, llevada a cabo mediante NEUROPSI atención y memoria y WAIS IV; de esta manera, los resultados se analizaron y se compararon con los datos de la evaluación pretratamiento para contrastar los cambios.

Cuarta fase, en ella se tomaron los datos o resultados finales de cada módulo, los cuales posteriormente fueron analizados a la luz de la estadística y las apreciaciones neuropsicológicas, para caso único, por medio de los siguientes índices de análisis que determinarían la magnitud del impacto terapéutico: el porcentaje de datos no solapados (PND) y el porcentaje de datos que exceden la mediana (PEM) (Sanz y García, 2015), los cuales determinaron de manera objetiva la magnitud del impacto terapéutico.

Debe tenerse en cuenta que, el paciente presentó un cuadro clínico claro, con compromiso atencional secundario a TCE frontal leve, donde se evidenció periodos de desconcentración frecuentes, dificultades con el sostenimiento, división y alternancia atencional y una evidente reducción de la capacidad de retención de información. 


\section{Método}

El presente estudio se orientó bajo lineamientos del paradigma de investigación cuantitativa, de acuerdo con las escalas de evaluación neuropsicológica, dado que estas arrojan datos cuantificables y objetivos; además, este paradigma permite definir variables, las cuales se determinaron como variable dependiente el desempeño neuropsicológico atencional del paciente, el cual sería susceptible a cambios que provoca la variable independiente, siendo en este caso la herramienta neurofeedback; así mismo, se estableció la hipótesis nula (refiere que el programa no tuvo efectividad) y la hipótesis de trabajo (indicaría que el programa tuvo efectividad), susceptibles de interpretación estadística (Hernández, Fernández y Baptista, 2014). Debe tenerse en cuenta como variable interviniente que, si bien el cerebro tiende a compensar las funciones que se afectan después de una lesión, esta compensación fisiológica espontánea dura determinado tiempo y puede verse muy beneficiada por distintas herramientas de estimulación, las cuales pueden optimizar la recuperación del paciente a nivel fisiológico y funcional (Garcés-Vieira, Suárez-Escudero, 2014).

La investigación se enmarca en un enfoque empírico-analítico, el cual busca dar una explicación del fenómeno ocurrido por medio de la observación y la experiencia; en este caso, a través del proceso de rehabilitación, de tal manera que los resultados obtenidos son sujeto de análisis para determinar la eficacia del programa (Ñaupas et al, 2014; Hernández et al, 2014).

\section{Técnicas e Instrumentos}

Se utilizó como instrumento una batería de exploración del circuito mnémico atencional (Ostrosky, Gómez, Matute, Rosselli, Ardila y Pineda, 2012) denominada NEUROPSI atención y memoria, la escala de inteligencia de Wechsler para adultos WAIS IV, para evaluar el nivel de Coeficiente intelectual (CI); la herramienta Neurofeedback, compuesta por cinco módulos (juegos o Brain games), dirigida a cada tipo de atención: atención sostenida, atención selectiva, control atencional, retención de información y atención dividida y el análisis 
estadístico para estudio de caso único que permite determinar la magnitud del cambio terapéutico, con las técnicas Porcentaje de datos no solapados (PND) desarrollado en 1998 por Scruggs y Mastropieri, y el Porcentaje de datos que exceden a la mediana (PEM) desarrollado por Hsen Ma en el año 2006 (Sanz y García, 2015).

\section{Resultados}

En la evaluación pretratamiento mediante la batería NEUROPSI, se encontró un bajo desempeño en funciones de atención selectiva, sostenida y control atencional. Los rangos de déficits y normalidad se exponen a continuación:

- 1-3 Severo

- 4-6 Leve a moderado

- 7-13 Normal

- 14-19 Normal alto

Tabla 1: Resultados atención y memoria

\begin{tabular}{|l|c|c|c|}
\hline \multicolumn{1}{|c|}{ Ítem } & $\begin{array}{c}\text { Puntuación normalizada } \\
\text { pretratamiento }\end{array}$ & $\begin{array}{c}\text { Puntuación normalizada } \\
\text { postratamiento }\end{array}$ & $\begin{array}{c}\text { Rango de } \\
\text { normalidad }\end{array}$ \\
\hline $\begin{array}{l}\text { Retención dígitos } \\
\text { progresión }\end{array}$ & 4 & 16 & $\begin{array}{c}4-6 \\
14-19\end{array}$ \\
\hline Cubos progresión & 5 & 10 & $\begin{array}{c}4-6 \\
7-13\end{array}$ \\
\hline Detección visual aciertos & 6 & 14 & $\begin{array}{c}4-6 \\
14-19\end{array}$ \\
\hline Detección de dígitos total & 9 & 12 & $7-13$ \\
\hline Series sucesivas & 8 & 13 & $7-13$ \\
\hline
\end{tabular}

En cuanto a la evaluación postratamiento, como se puede ver en la tabla 1, las puntuaciones fueron significativas, en especial, con los tres primeros ítems que indican un desempeño funcional normal alto (14-19). 
Tabla 2: Resultados memoria de trabajo

\begin{tabular}{|l|c|c|c|}
\hline \multicolumn{1}{|c|}{ Ítem } & $\begin{array}{c}\text { Puntuación normalizada } \\
\text { Pretratamiento }\end{array}$ & $\begin{array}{c}\text { Puntuación normalizada } \\
\text { Postratamiento }\end{array}$ & Rango \\
\hline $\begin{array}{c}\text { Retención dígitos } \\
\text { regresión }\end{array}$ & 4 & 10 & $4-6$ \\
\hline Cubos Gresión & 7 & 12 & $7-13$ \\
\hline
\end{tabular}

Otros de los hallazgos del NEUROPSI expuestos en la tabla 2, se obtuvieron con las subpruebas que evalúan memoria de trabajo; estos resultados postratamiento evidenciaron, según escala de valoración utilizada, un desempeño normal a nivel de estos procesos rango (7-13).

Tabla 3: Resultados coeficiente intelectual

\begin{tabular}{|l|c|c|c|}
\hline \multicolumn{1}{|c|}{ Ítem } & $\begin{array}{c}\text { Puntuación } \\
\text { pretratamiento }\end{array}$ & $\begin{array}{c}\text { Puntuación post } \\
\text { tratamiento }\end{array}$ & $\begin{array}{c}\text { Rango de } \\
\text { normalidad }\end{array}$ \\
\hline Índice de comprensión verbal & 100 & 114 & $90-115$ \\
\hline Índice de razonamiento perceptual & 101 & 105 & $90-115$ \\
\hline Índice memoria de trabajo & 97 & 102 & $90-115$ \\
\hline $\begin{array}{l}\text { Índice de velocidad de } \\
\text { procesamiento de información }\end{array}$ & 89 & 102 & $90-115$ \\
\hline Coeficiente intelectual total & 100 & 107 & $90-115$ \\
\hline
\end{tabular}

Por otro lado, se encuentran los resultados WAIS IV que se presentan en la tabla 3, en la que es posible observar cambios significativos en los ítems entre pretratamiento y postratamiento, principalmente, como en el índice de velocidad de procesamiento de información, en este ítem el alcance fue de 102 puntos, consecuentemente a la intervención con neurofeedback.

A continuación, se presentan los resultados del análisis estadístico para estudio de caso único, con el cual se determinó la magnitud del impacto terapéutico (mejoría del proceso atencional). 
Tabla 4: Valores convencionales para interpretar la magnitud de cambio terapéutico

\begin{tabular}{|l|l|l|l|}
\hline \multicolumn{1}{|c|}{ Índice } & \multicolumn{1}{|c|}{ Porcentaje } & \multicolumn{1}{|c|}{ Rango de efectividad } & \multicolumn{1}{|c|}{ Referencia } \\
\hline \multirow{4}{*}{ PND } & $<50 \%$ & Tratamiento no efectivo & \multirow{2}{*}{ Scruggs y Mastropieri (1998) } \\
\cline { 2 - 4 } & $50 \%-69 \%$ & Efectividad cuestionable & \\
\cline { 2 - 4 } & $70 \%-89 \%$ & Tratamiento bastante efectivo & \\
\cline { 2 - 4 } & $>90 \%$ & Tratamiento muy efectivo & \multirow{2}{*}{ Ma (2006) } \\
\hline \multirow{2}{*}{ PEM } & $<70 \%$ & Tratamiento cuestionable o no efectivo & \\
\cline { 2 - 4 } & $70 \%-89 \%$ & Tratamiento moderadamente efectivo & \\
\cline { 2 - 4 } & $90 \%-100 \%$ & Tratamiento muy efectivo & \\
\hline
\end{tabular}

Nota: Cuadro de clasificación tomada de Sanz y García (2015).

Para comprender el rango de efectividad del tratamiento de forma clara, se expone la tabla 4, sobre valores convencionales para interpretar la magnitud del cambio terapéutico, de acuerdo con el índice (porcentaje de datos no solapados PND y el porcentaje de datos que exceden la mediana PEM) y su respectivo porcentaje, el que determina el rango de efectividad.

Tabla 5: Resultados PND del tratamiento

\begin{tabular}{|l|c|}
\hline \multicolumn{1}{|c|}{ Proceso atencional } & PND \\
\hline Atención sostenida & 91,7 \\
\hline Atención selectiva & 91,7 \\
\hline Control atencional & 91,7 \\
\hline Memoria a corto plazo y retención de información & 83,3 \\
\hline Atención dividida & 83,3 \\
\hline
\end{tabular}

$P N D$. Con el respecto al primer índice los resultados de la tabla 5, indican un desempeño en atención sostenida del $91,7 \%$, atención selectiva y control atencional y un 83,3 \% en retención de información-memoria a corto plazo y atención dividida. 
Tabla 6: Resultados PEM

\begin{tabular}{|l|l|}
\hline \multicolumn{1}{|c|}{ Proceso atencional } & \multicolumn{1}{c|}{ PEM } \\
\hline Atención sostenida & 91,67 \\
\hline Atención selectiva & 100 \\
\hline Control atencional & 75 \\
\hline Memoria a corto plazo y retención de información & 75 \\
\hline Atención dividida & 25 \\
\hline
\end{tabular}

PEM. Los resultados que se muestran en la tabla 6, con el índice PEM evidenciaron un desempeño del 91,67\% para atención sostenida, un $100 \%$ en atención selectiva, un $75 \%$ obtenido en control atencional y retención de información-memoria a corto plazo y atención dividida con un $25 \%$.

\section{Discusión}

El presente estudio tuvo como objetivo comprobar la efectividad de un programa de rehabilitación atencional mediante neurofeedback. Se encontró durante la evaluación pretratamiento alteraciones principalmente en procesos psicológicos como atención sostenida, selectiva y control atencional, y otras dificultades a nivel de retención de información, algunos déficits en memoria de trabajo y velocidad de procesamiento de información. Dichos procesos mostraron una mejoría significativa después de la intervención mediante neurofeedback, los cuales se evidencian en los resultados postratamiento.

Estos hallazgos son concordantes con los postulados de González, Giraldo, Ramírez y Quijano (2012) quienes manifiestan que, una rehabilitación del proceso atencional puede generar cambios positivos que permitan mejorar la funcionalidad del paciente, gracias a la rehabilitación neurocognitiva. Así, la rehabilitación dirigida a procesos afectados permitió que el paciente, mediante estas herramientas tecnológicas, mejore considerablemente el desempeño de los componentes atencionales afectados.

Puntualizando en los hallazgos, se encontró que a nivel de atención selectiva el paciente logró optimizar su capacidad de respuesta, en cuanto a la 
discriminación de estímulos e inhibición de respuestas irrelevantes o distractores. Este hallazgo se relaciona con los planteamientos de Cuervo, Rincón y Quijano (2009), quienes observaron que, a nivel de atención selectiva, los sujetos tuvieron una mejor capacidad para seleccionar e inhibir la información del entorno. Frente a este resultado, es posible determinar que mediante la aplicación de la herramienta neurofeedback, fue posible apoyar en gran medida la mejoría del proceso atencional afectado.

Otra de las funciones mayormente beneficiadas tiene que ver con la atención sostenida, puesto que el paciente logró optimizar el tiempo de respuesta en tareas continuadas, y la amplitud en cuanto al tiempo en que logra estar concentrado. Estos resultados se pueden comparar con hallazgos evidenciados por Bonilla et al (2016), quienes lograron como resultado de sus investigaciones, cambios positivos en el tono atencional de sus pacientes después de una rehabilitación cognitiva. Asimismo, los resultados de Brocalero y Pérez (2011), también son congruentes con los hallazgos del presente estudio, en cuanto a la mejoría de la capacidad de focalización atencional, logrando una mejor y más amplia concentración.

Así mismo, los hallazgos y alcances muestran que ha sido posible revertir los daños de otras funciones cognitivas relacionadas, como la memoria de trabajo y algunos componentes de las funciones ejecutivas como secuenciación, y fluidez verbal y no verbal, componentes que se vieron en decremento durante la evaluación pretratamiento, y que acompańaban el déficit atencional. Lo anterior se argumenta claramente dada la observación del desempeño del paciente en diversas tareas atencionales, la ilación de su discurso y su testimonio de mejoría, relacionado con una mayor funcionalidad y desempeño cognitivo; acorde con hallazgos de autores como Ríos, Muñoz y Paul (2007); Carvajal, Henao, Uribe, Giraldo y Lopera (2009); García, Gómez, Rodríguez, Sánchez, Zumarraga, Enseñat, Bernabeu, Tormos y Roig (2010) Quijano y Cuervo (2011), Moraleda, Romero y Cayetano, (2011) y Bonilla et al (2016). Además, se indica que las funciones anteriormente mencionadas se encuentran estrechamente relacionadas y una rehabilitación de tipo atencional puede generar mejoría en otros procesos, debido a la estrecha funcionalidad de las redes atencionales, velocidad de procesamiento y procesos de memoria (Bernabéu, 2017). 
Con base en los anteriores resultados, es posible mencionar que el neurofeedback ha demostrado ser efectivo en la rehabilitación atencional, mejorando la capacidad en la función atencional del paciente; como lo aseguran Fernández et al (2012); Young, Tae \& Hwa, (2015); Kianbakht et al, (2015) y Fajardo y Guzmán (2016) que el tratamiento con herramientas tecnológicas vanguardistas como esta, influyen en la optimización de la actividad de las distintas ondas cerebrales, por medio de la retroalimentación y la autorregulación de procesos atencionales, de lo cual se encarga el neurofeedback que se va constituyendo como herramienta efectiva para la rehabilitación de las consecuencias cognitivas del TCE.

El neurofeedback se adapta a las necesidades del paciente, teniendo una interfaz amable, una forma de uso sencilla y un aumento progresivo en su complejidad, permitiendo un enganche efectivo; características que permiten el logro de metas, como el aumento en la puntuación del coeficiente intelectual o mejorar la capacidad de aprendizaje. Además de ser una técnica no invasiva, es una herramienta efectiva para cumplir con objetivos como la restitución de los procesos afectados que ayudan a mejorar el desempeńo cognitivo.

Finalmente, el análisis estadístico que midió la magnitud del cambio terapéutico evidenció que el tratamiento es bastante efectivo, según los resultados estadísticos, principalmente a nivel de atención sostenida, selectiva y control atencional, analizados a partir de la significancia estadística. Los resultados expuestos por Sanz y García (2015), refieren que los índices permiten tener una comprensión objetiva en los resultados de un estudio de caso, indicando que existe un cambio estadísticamente significativo, considerando que es pertinente tener en cuenta otras áreas de la vida del paciente.

De lo anterior se deduce que, si bien los resultados demuestran un nivel significativo estadísticamente en el tratamiento, es posible también corroborarlo con los datos observables de las evaluaciones pre y postratamiento, estos muestran una clara evidencia de mejoría en el nivel de funcionamiento cognitivo (Sanz y García, 2015). 


\section{Conclusiones y recomendaciones}

La técnica de neurofeedback utilizada en el presente estudio se enfocó en la proliferación de conectividad neuronal y actividad de ondas bioeléctricas, relacionadas con la función atencional prevalentemente en los lóbulos frontales. Como resultado del estudio se observó una mejoría principalmente en atención selectiva, sostenida y foco atencional, posterior al proceso de estimulación con neurofeedback. Esto comprueba la efectividad de la técnica.

También es evidente la mejoría en otros procesos como velocidad de procesamiento, memoria de trabajo y funciones ejecutivas, lo cual es plenamente argumentable si se tiene en cuenta la relación que estos procesos guardan con la atención; el aumento de la proliferación conectiva y la actividad de ondas bioeléctricas relacionadas con la atención — procesos neuro anatómicamente similares a nivel de ubicación-, también se vieron beneficiados.

Los índices PND y PEM evidenciaron significancia estadística positiva en relación con la efectividad de la estimulación con neurofeedback, lo cual se corroboró con el mejoramiento del funcionamiento cognitivo prevalentemente atencional del paciente.

Se recomienda a los psicólogos y neuropsicólogos el uso del neurofeedback en los casos de TCE y posiblemente otro tipo de lesiones y patologías cerebrales que comprometan la función atencional, dado que se ha comprobado que las herramientas de biorretroalimentación — neurofeedback — son efectivas en los procesos de rehabilitación neuropsicológica de funciones cognitivas.

Finalmente, es de suma importancia que los profesionales de la salud mental que trabajan con este tipo de casos revisen nuevas técnicas y herramientas tecnológicas que potencialicen la efectividad de sus procesos, haciendo control permanente de los mismos y midiendo su efectividad con fórmulas estadísticas precisas para este fin, como la usada en este estudio. 


\section{Referencias}

Bárcena, A., Rodríguez, C., Rivero, B., Cañizal, J., Mestre, C., Calvo, J., Molina, A., y Casado, J. (2006). Revisión del Traumatismo craneoencefálico. Neurocirugía, 17, 495-518. Recuperado de http://scielo.isciii.es/pdf/neuro/v17n6/1.pdf

Barrera, L., Gómez, E., y Prieto, L. (2013). Efectividad del tratamiento con Neurofeedback en trastornos del estado de ánimo, ansiedad y fibromialgia: una revisión de literatura. (Trabajo de Grado). Universidad del Rosario. Recuperado de https:// repository.urosario.edu.co/bitstream/handle/10336/4458/BarreraFerro-Laura-2013.pdf? sequence $=1$ \&isAllowed $=y$

Barrero, C., García, S., y Ojeda, A. (2005). Índice de Barthel (IB): un instrumento esencial para la evaluación funcional y la rehabilitación, plasticidad y restauración neurológica. Nuevos Horizontes, 4(1-2): 81-85. Disponible en https://bit. ly/2jZBz66

Bernabéu, E. (2017). La atención y la memoria como claves del proceso de aprendizaje. Aplicaciones para el entorno escolar. ReiDoCrea, 6(2), 16-23. doi: 00000001-9724-7138

Bernal, O., Vega, M., y Hernández, J. (2009). Consecuencias neuropsiquiátricas del trauma craneoencefálico. Revista Med, 17(1), 65-74. Disponible en https://bit. ly/2lEcX79

Bonilla, J., Gonzáles, A., Amaya, E., Ríos, A., y Bonilla, G. (2016). Resultados de un programa de rehabilitación neurocognitiva en pacientes con secuelas de trauma craneoencefálico. Revista Chilena de neuro-psiquiatría, 54(2), 113-122. http:// dx.doi.org/10.4067/S0717-92272016000200005

Brocalero, A., y Pérez, Y. (2011). Proceso de rehabilitación cognitiva en un caso de traumatismo craneoencefálico. Clinica Contemporánea, 2(2), 177-185. DOI https://doi.org/10.5093/cc2011v2n2a5

Camelo, S., Rojas, D., Mejía, A., y Castro, R. (2015). Registro de la actividad eléctrica cerebral de la atención implicada en la conducción bajo el efecto del alcohol usando un instrumento BCI (Brain Control Interface). Revista Diversitas. Perspectivas en Psicología, 11(2), 217-233. http://dx.doi.org/10.15332/s17949998.2015.0002.04

Carvajal-Castrillón, J., Henao, E., Uribe, C., Giraldo, M., y Lopera, F. (2009). Rehabilitación cognitiva en un caso de alteraciones neuropsicológicas y funcionales por traumatismo craneoencefálico severo. Revista Chilena de Neuropsicología, 4(1), 52-63. Disponible en https://bit.ly/2k46Zfn 
Cuervo, M., Rincón, A., Y Quijano, M. (2009). Efecto de un programa de intervención en atención para pacientes con trauma craneoencefálico moderado. Revista Diversitas, 5(2), 361-371. Disponible con https://bit.ly/2lDgNgO

Donéstevez, A., Cañizares O., Alba, C., y Alegret, M. (2017). Caracterización neuropsicológica de pacientes con trauma craneoencefálico frontal leve y moderado. Revista Acta Médica del Centro, 11(2), 4-9. Disponible en https://bit. ly/2k4rWH4

Fajardo, A., y Guzmán A. (2016). Neurofeedback, aplicaciones y eficacia. Interdisciplinaria, 33(1), 81-93. Disponible en https://bit.ly/2m3h3Gb

Fernández, E., Bringas, M., Salazár, S., Rodríguez, D., García, M., \& Torres, M. (2012). Clinical Impact of RehaCom software for cognitive rehabilitation of patients with acquired brain injury, MEDICC Review, 14(4), 32-35. Disponible en https://bit.ly/2kxW8L0

Garcés-Vieira, M., y Suárez-Escudero, J. (2014). Neuroplasticidad: aspectos bioquímicos y neurofisiológicos. Rev CES Med, 28(1), 119-132. Disponible en https://bit.ly/2CKma5h

García-Molina, A., Gómez, A., Rodríguez, P., Sánchez-Carrión, R., Zumarraga, L., Enseñat, A., Bernabeu, M., Tormos, J., y RoigRovira, T. (2010). Programa de telerehabilitación cognitiva en el traumatismo craneoencefálico. Institut Universitari de Neurorehabilitación Guttmann. Fundación MAPFRE, 21(1), 58-63. Disponible en https://bit.ly/2kxWJMK

González, D., Giraldo, C., Ramírez, D., y Quijano, M. (2012). Cambios en la calidad de vida en pacientes con trauma craneoencefálico severo después de un programa de rehabilitación. Psychol. av. Discip, 6(1), 77-89. Disponible en https://bit. ly/2kzvOAb

Guzmán, F. (2008). Fisiopatología del trauma craneoencefálico. Colombia Médica, 39, 78-84. Disponible en https://bit.ly/2ImH8Wz

Hernández, S., Fernández, C., y Baptista, P. (2014). Metodología de la investigación. México: McGraw Hill.

Juárez, J., y Machinskaya, R. (2013). Rehabilitación Neuropsicológica de un caso de lesión fronto-temporal derecha como resultado de un traumatismo craneoencefálico. Revista Neuropsicología Latinoamericana, 5(1), 28-31. doi:10.5579/ $\operatorname{rnl} .2013 .0113$

Kianbakht, M., Naghel, S., Alidadi, F., Nejati, V., Kohandel, H., Ansarini, G., \& Namdari, V. (2015). Effectiveness of Neurofeedback Associated with Cognitive 
Rehabilitation Therapy on Children with Attention Defect Hyperactivity Disorder (ADHD). The International Journal Indian Psychology, 2(4), 18-29. DIP: B00302V2I42015. Disponible en https://bit.ly/2lIPBNB

Lubrini, G., Periáñez, J., y Ríos-Lago, M. (2009). Estimulación cognitiva y rehabilitación neuropsicológica de la atención. Universitat Oberta de Catalunya: FUOC P09/80548/00292. Disponible en https://bit.ly/2lCLTFy

Machuca, F., León, J., y Barroso, J. (2006). Eficacia de la rehabilitación neuropsicológica de inicio tardío en la recuperación funcional de pacientes con daño cerebral traumático. Revista Española de Neuropsicología, 8(3-4), 81-103.

Méndez, A., (2011). Dimensiones de la función ejecutiva en trauma craneoencefálico por accidente de tránsito. Bogotá: Universidad de San Buenaventura. Disponible en https://bit.ly/2SA2lpw

Méndez, O., y Ordoñez, G. (2012). Evaluación Neuropsicológica en el Traumatismo Craneoencefálico Severo: Estudio de caso. México: Benemérita Universidad Autónoma de Puebla.

Moraleda, E., Romero, M., y Cayetano, M. (2011). Perfil cognitivo de los pacientes con traumatismo craneoencefálico grave: Un estudio a los dos ańos de evolución, Revista Chilena de Neuropsicología, 6(1), 41-47. Disponible en https://bit. $\mathrm{ly} / 2 \mathrm{kxg} 3 \mathrm{cY}$

Ñaupas, H., Mejía, E., Novoa, E., y Villagómez, A. (2014). Metodología de la investigación, Cuantitativa - Cualitativa y Redacción de Tesis. 4ta Edición. Bogotá: Ediciones la U.

Organización Mundial de la Salud (OMS). (2016). Road traffic injuries. Ginebra, Suiza. Disponible en: https://bit.ly/19zLNCQ

Ostrosky, F., Gómez, M., Matute, E., Rosselli, M., Ardila, A., y Pineda, D. (2012). Neuropsi. Atención y Memoria. 2da Ed. Manual Moderno.

Quijano M., y Cuervo, M. (2011). Alteraciones Cognoscitivas después de un trauma craneoencefálico. Acta Colombiana de Psicología, 14(1), 71-80. Disponible en https://bit.ly/2k4vx82

Quijano, M., Arango, J., Cuervo, M., y Aponte, M. (2012). Neuropsicología del Trauma Craneoencefálico en Cali, Colombia. Revista Ciencia Salud, 10(1), 2131. Disponible en https://bit.ly/2k7jgjl

Ríos, M., Muñoz, J., y Paúl, N. (2007). Alteraciones de la atención tras daño cerebral traumático: evaluación y rehabilitación. Revista de Neurología, 44(5), 291-297. DOI: $10.33588 / \mathrm{rn} .4405 .2006208$ 
Sanz J., y García-Vera, M. (2015). Técnicas para el análisis de diseños de caso único en la práctica clínica: ejemplos de aplicación en el tratamiento de víctimas de atentados terroristas. Universidad Complutense de Madrid, España. Elsevier, 26(3), 167-180. http://dx.doi.org/10.1016/j.clysa.2015.09.004

Stokes, D., \& Lappin, M. (2010). Neurofeedback and biofeedback with 37 migraineurs: a clinical outcome study. Behavioral and Brain Functions, 6(9). https:// doi.org/10.1186/1744-9081-6-9

Young Cho H., Tae Kim K., \& Hwa Jung. (2015). Effects of computer assisted cognitive rehabilitation on brain wave, memory and attention of stroke patients: randomized control trial. Journal. Psychology. Ther. Sci., 27(4), 1029-1032. doi:10.1589/jpts.27.1029 